Article

\title{
Effect of Carbon on the Volume of Solid Iron at High Pressure: Implications for Carbon Substitution in Iron Structures and Carbon Content in the Earth's Inner Core
}

\author{
Jing Yang ${ }^{1, *}$, Yingwei Fei ${ }^{1}$, Xiaojun $\mathrm{Hu}^{2}$, Eran Greenberg ${ }^{3}\left[\right.$ and Vitali B. Prakapenka ${ }^{3}$ \\ 1 Geophysical Laboratory, Carnegie Institution of Washington, 5251 Broad Branch Road, N.W., Washington, \\ DC 20015, USA; yfei@carnegiescience.edu \\ 2 School of Science, Wuhan University of Technology, Wuhan 430070, China; xjhu@whut.edu.cn \\ 3 Center for Advanced Radiation Sources, University of Chicago, Chicago, IL 60637, USA; \\ erangre@gmail.com (E.G.); prakapenka@cars.uchicago.edu (V.B.P.) \\ * Correspondence: jyang@carnegiescience.edu
}

Received: 15 October 2019; Accepted: 16 November 2019; Published: 20 November 2019

check for updates

\begin{abstract}
Understanding the effect of carbon on the density of hcp (hexagonal-close-packed) Fe-C alloys is essential for modeling the carbon content in the Earth's inner core. Previous studies have focused on the equations of state of iron carbides that may not be applicable to the solid inner core that may incorporate carbon as dissolved carbon in metallic iron. Carbon substitution in hcp-Fe and its effect on the density have never been experimentally studied. We investigated the compression behavior of $\mathrm{Fe}-\mathrm{C}$ alloys with 0.31 and $1.37 \mathrm{wt} \%$ carbon, along with pure iron as a reference, by in-situ X-ray diffraction measurements up to $135 \mathrm{GPa}$ for pure Fe, and $87 \mathrm{GPa}$ for Fe-0.31C and $109 \mathrm{GPa}$ for Fe-1.37C. The results show that the incorporation of carbon in hcp-Fe leads to the expansion of the lattice, contrary to the known effect in body-centered cubic (bcc)-Fe, suggesting a change in the substitution mechanism or local environment. The data on axial compressibility suggest that increasing carbon content could enhance seismic anisotropy in the Earth's inner core. The new thermoelastic parameters allow us to develop a thermoelastic model to estimate the carbon content in the inner core when carbon is incorporated as dissolved carbon hcp-Fe. The required carbon contents to explain the density deficit of Earth's inner core are 1.30 and $0.43 \mathrm{wt} \%$ at inner core boundary temperatures of $5000 \mathrm{~K}$ and $7000 \mathrm{~K}$, respectively.
\end{abstract}

Keywords: earth's core; light elements; interstitial carbon; X-ray diffraction

\section{Introduction}

Carbon $(\mathrm{C})$ has been considered as one of the light elements to account for the density deficit of the Earth's core based on emerging evidence from cosmological, seismic, geochemical, and mineral physics data [1-4]. If the Earth's inner core were crystallized from a carbon-bearing core, either iron (Fe) carbide or Fe with interstitial carbon would be a candidate for the solid inner core. Whether the inner core crystallizes into an Fe carbide or Fe with interstitial carbon phase depends on the eutectic composition at inner core boundary (ICB) as well as the bulk carbon content in the Earth's core $[2,5,6]$. For an Fe carbide core, the $\mathrm{Fe}_{7} \mathrm{C}_{3}$ phase has been proposed as a leading candidate because its properties at high pressure can reconcile with several seismic observations, including matching the observed density, sound velocity and Poisson's ratio $[4,7,8]$. On the other hand, thermodynamic calculations of the Fe-C system at the ICB condition based on experimental data at high pressure suggest that if the 
bulk carbon content is less than $2.24 \mathrm{wt} \%$ in the core, a metallic Fe core with interstitial carbon, instead of Fe carbide, would be expected [2].

Interstitial carbon under solubility limit in its host lattice would not change the crystal structure of the host metal. Although it is still debated that whether Fe in the inner core would be in a body-centered cubic (bcc) $[9,10]$ or hexagonal close-packed structure (hcp), the hcp-Fe is commonly accepted as the stable phase in the core [11-13]. The elastic properties of hcp-Fe can also provide a plausible explanation for the observed elastic anisotropy of the inner core [14,15]. The interstitial carbon in Fe would have an effect on the density and elastic properties of Fe at high pressure. Theoretical results show that interstitial carbon in hcp-Fe increases the compressional wave velocity, decreases the shear wave velocity, with enhanced seismic anisotropy. Furthermore, only $1-2.5 \mathrm{wt} \%$ of carbon is needed to match the density deficit of the inner core depending on the geotherm profile of the core [16]. However, there have been no experimental data on the effect of carbon on the density and elastic properties of hcp-Fe at high pressure. The current modeling of the Fe-C phase diagram at high pressure assumes that the behavior of carbon in the hcp-Fe structure is similar to that in the bcc- or fcc-Fe [2,5]. Such an assumption needs to be further examined by experiments in the stability field of hcp-Fe. Recent melting experiments on the Fe-C binary system showed that carbon content in the solid Fe remains 1 wt $\%$ up to $255 \mathrm{GPa}$ [6]. The authors estimated the density of Fe with $1 \mathrm{wt} \%$ carbon by assuming a linear density change between pure $\mathrm{Fe}, \mathrm{Fe}_{3} \mathrm{C}$ and $\mathrm{Fe}_{7} \mathrm{C}_{3}$ because of the lack of data on the density of $\mathrm{Fe}$ with interstitial carbon. Such a linear interpolation could lead to a large uncertainty in the estimated carbon content.

In this study, we directly determined the density of Fe with dissolved carbon at high pressure to evaluate the relationship between the carbon content and the inner core density deficit. We have determined the equations of state of pure Fe and two Fe-C alloys with 0.31 and 1.37 wt \% interstitial carbon up to $135 \mathrm{GPa}$. The experiments with multiple samples in the same sample chamber under the neon pressure medium provide high precision data that allow systematic evaluation of the carbon effect on the elastic properties and density of hcp-Fe. Using the new data together with previously published thermoelastic parameters for hcp-Fe, we placed constraint on the maximum carbon content in the inner core by comparing the density profiles between our mineral physics model and the seismic observation.

\section{Experimental Method}

\subsection{Sample Synthesis}

We used the commercial standard reference materials from National Institute of Standards and Technology (NIST), 19H basic electric steel (Fe with 0.2 wt \% carbon, Fe-0.2C) and 5m Cast Iron (Fe with 2.59 wt \% carbon, Fe-2.59C), and pure Fe powder (Alfa Aesar, Haverhill, MA, USA, 99.5\%) as starting materials for the synthesis of Fe-C alloys with $0.31 \mathrm{wt} \%$ carbon (denoted as Fe-0.31C) and 1.37 wt \% carbon (denoted as Fe-1.37C). It should be noted that the carbon content in the commercial NIST reference materials were measured in bulk, and they were not homogeneous in the micron scale, which needed further high pressure-temperature treatments. The Fe-0.31C sample was homogenized and sintered from the NIST Fe-0.2C, while Fe-1.37C sample was synthesized from mixed pure Fe and Fe-2.59C with a 1:1 ratio. The starting materials were loaded into an $\mathrm{MgO}$ capsule with crushable $\mathrm{MgO}$ spacers and sintered using piston cylinder apparatus at $1 \mathrm{GPa}$ and $1150{ }^{\circ} \mathrm{C}$ at the Geophysical Laboratory. The temperature was measured with a type-C (W-5\%Re vs. W-26\%Re) thermocouple for all the experiments. Samples were heated at a rate of $100{ }^{\circ} \mathrm{C} / \mathrm{min}$ to the set temperature and kept for at least 24 hours to ensure the sample homogeneity.

\subsection{Carbon Analytical Procedure}

The synthesized samples with a cylinder shape were cut into two pieces using a diamond saw. For each sample, one of the pieces was mounted into resin epoxy and polished with $0.05 \mu \mathrm{m} \mathrm{Al}_{2} \mathrm{O}_{3}$ gel on a lapidary wheel using water as lubricant. After a desirable polished surface was achieved, samples 
were cleaned using ethanol and then coated with Ir with a thickness of $100 \mathrm{~nm}$. The samples were analyzed using electron probe microanalyzer (EMPA, JEOL 8530F, Akishima, Tokyo) at the Geophysical Laboratory with $12 \mathrm{keV}$ accelerating voltage and $30 \mathrm{nA}$ current. The EMPA is equipped with an LDE6L crystal for high-precision carbon analysis. We also employed cold finger for all the carbon analyses, which reduced the carbon contamination in the vacuum chamber by half compared to that of without cold finger $[17,18]$. The standards were also coated with Ir with a thickness of $100 \mathrm{~nm}$ to keep X-ray absorptions uniform. The on-peak time was 10 second and off-peak time was $5 \mathrm{~s}$ for the carbon analysis. NIST pure $\mathrm{Fe}$, synthetic $\mathrm{Fe}_{3} \mathrm{C}(6.7 \mathrm{wt} \% \mathrm{C})$ and $\mathrm{Fe}_{7} \mathrm{C}_{3}(8.43 \mathrm{wt} \% \mathrm{C})$ were used as standards for carbon. The typical peak to background ratio for the $\mathrm{C} K \alpha$ was about 14.

The $\mathrm{Fe}_{3} \mathrm{C}$ standard was synthesized in a piston cylinder apparatus using a half inch assembly with $\mathrm{BaCO}_{3}$ pressure cell, crushable $\mathrm{MgO}$ spacers, and graphite furnace at the Geophysical Laboratory. A pure Fe wire ran in presence of excess carbon was loaded in a graphite capsule and kept at 2 GPa and $1200{ }^{\circ} \mathrm{C}$ for $51 \mathrm{~h}$. The $\mathrm{Fe}_{7} \mathrm{C}_{3}$ standard was synthesized using a multi-anvil apparatus at the University of Michigan [8], with the mixture of fine Fe and excess graphite powers loaded into an $\mathrm{MgO}$ capsule and heated to $1200{ }^{\circ} \mathrm{C}$ at $14 \mathrm{GPa}$ for $7 \mathrm{~h}$.

\subsection{Diamond Anvil Cell and Synchrotron X-ray Diffraction Experiements}

For X-ray diffraction (XRD) experiments, high-pressure conditions up to 135 GPa were generated using a symmetric diamond anvil cell (DAC) with a pair of $350 \mu \mathrm{m}$ flat or 150-250 $\mu \mathrm{m}$ beveled culet diamonds. The bulk samples were cut into pieces with dimensions of approximately $25 \mu \mathrm{m} \times 30 \mu \mathrm{m} \times$ $7 \mu \mathrm{m}$ or $10 \mu \mathrm{m} \times 10 \mu \mathrm{m} \times 5 \mu \mathrm{m}$ using a tungsten carbide knife. Rhenium gaskets were pre-indented to a thickness of $40 \mu \mathrm{m}$ or $25 \mu \mathrm{m}$ using the opposing diamonds. Holes of $180 \mu \mathrm{m}$ or $80 \mu \mathrm{m}$ were drilled in the center of the pre-indented area. Three samples (pure Fe, Fe-0.31C, and Fe-1.37C) were loaded in the sample chamber, separated with spaces filled with neon pressure medium. A small Au flake was also loaded as a pressure calibrant. Neon served as an additional pressure calibrant and a hydrostatic pressure medium [19].

Angle-dispersive synchrotron XRD measurements were performed at 13-IDD GSECARS beamline at the Advanced Photon Source (APS), Argonne National Laboratory (ANL). The incident X-ray beam had a monochromatic wavelength of $0.3344 \AA$ and a full width at half maximum of $5 \mu \mathrm{m} \times$ $5 \mu \mathrm{m}$. At each pressure point, the X-ray diffraction images of pure Fe, Fe-0.31C, Fe-1.37C and Au were recorded for an exposure time of 20-50 s. Data were collected at an interval of 1-2 GPa up to $45 \mathrm{GPa}$ for the first run (1st RUN) and 10-15 GPa interval up to $135 \mathrm{GPa}$ for the second run (2nd RUN). The XRD images were integrated using the software Dioptas [20]. The diffraction patterns were analyzed using the Crystallography Data Analysis software (GSAS) [21] to obtain the lattice parameters at different pressures.

\section{Results}

Iron adopts different packing types upon heating or compression: bcc at ambient condition, fcc upon heating, and hcp upon compression. The speciation of carbon in Fe depends on carbon concentration, pressure, and temperature. The carbon solubility in the bcc-Fe was relatively low compared to that of the case in fcc-Fe due to the large distance between Fe atoms. The maximum carbon solubility in bcc-Fe was less than $\sim 0.022 \mathrm{wt} \%$ at ambient pressure and temperature. In fcc-Fe, the maximum carbon solubility was as high as $2.14 \mathrm{wt} \%$ at ambient pressure [22], and slightly decreased with increasing pressure [23]. For example, at $1 \mathrm{GPa}$ and $1150{ }^{\circ} \mathrm{C}$, which was the synthesis condition in this study, the maximum carbon dissolved in fcc-Fe is $\sim 2.0 \mathrm{wt} \%$. By a rapid quenching of carbon dissolved fcc-Fe, the carbon stayed in the interstitial site of the lattice to form a bct (body-centered tetragonal) structure, which is a deformed bcc structure [24]. In order to obtain a homogeneous sample with a high carbon concentration, the quenched samples with a bct structure were synthesized and loaded into the diamond anvil cells for further high-pressure experiments. In this study, the results 
showed that the bct structure transformed to hcp structure at 14-16 GPa at room temperature, which was slightly higher than the pressure of the bcc to hcp phase transition.

Back scattered electron (BSE) images of our polished samples (Figure S1) showed chemical homogeneity without any quench textures for the entire recovered sample, which was important to extract a tiny sample with the same composition for the DAC experiments. The inhomogeneous samples and samples with quenched texture as shown in BSE images were excluded from further analysis and DAC experiments (Figure S2). We randomly sampled at least 40 points on each sample for the carbon analysis. With the insertion of the cold finger, measurements of the pure Fe standard for carbon always yielded $\sim 0.2 \mathrm{wt} \%$ carbon, which was taken as the background and corrected accordingly for the carbon analysis of the alloys. The background corrected counts for the carbon K $\alpha$ peak showed a linear relationship with the concentrations of carbon in the standards. We used this calibration curve to determine the carbon contents in the samples, yielding $0.31( \pm 0.03) \mathrm{wt} \%$ and $1.37( \pm 0.06) \mathrm{wt} \%$ carbon for the synthesized samples used in the DAC experiments (Figure S1).

We loaded pure Fe, Fe-0.31C, and Fe-1.37C into one sample chamber to obtain high-precision data for comparison. XRD patterns were measured for each sample with a 1-2 GPa interval in the first run. The XRD patterns for the C-bearing samples were consistent with a bct-Fe structure based on peaks broadening comparing to those of pure Fe (Figure 1c). The bct structure was a slightly distorted bcc structure with a diverged $c$ axis. It is usually difficult to measure the $c$ axis from powder diffraction data because of peak overlap [24]. Figure la shows the (110) peak of bcc phase for pure Fe (Fe-OC), $\mathrm{Fe}-0.31 \mathrm{C}$, and Fe-1.37C at 2.3 GPa, which had the highest relative peak intensity in the XRD pattern. In Figure 1a,c, the intensity of each spectrum was normalized to one to better compare the peak shifts. Furthermore, the full XRD patterns are shown in Figure 1c,d. Generally, the peaks shifted to the higher angle side with increasing pressure and reducing lattice parameters. We only calculated the lattice constant $a$ for bct-Fe using (110) and (200) peaks since other peaks used to calculate $c$ axis were difficult to be identified (Figure 1c). At $2.3 \mathrm{GPa}$, comparing the peak positions of $\mathrm{Fe}$ (Fe-0C), Fe-0.31C, and Fe-1.37C, the Fe-1.37C had the smallest $a$, and Fe-0C - the largest $a$ lattice parameter (Figure 1a). This trend continued up to $14.4 \mathrm{GPa}$ before the hcp phase transition (Figure S3).

We observed the structure transformation in Fe-0C, Fe-0.31C, and Fe-1.37C to hcp structure started at $\sim 16 \mathrm{GPa}$ and was completed at $\sim 20 \mathrm{GPa}$. Figure $1 \mathrm{~b}$ shows the (002) and (101) peaks of iron in its hcp structure for Fe-0C, Fe-0.31C and Fe-1.37C at $32.5 \mathrm{Gpa}$. The (111) peak of solid neon is also shown. We used the neon (111) peak as a primary pressure calibrant above 16 GPa in order to compare the subtle difference in pressure within the sample chamber. With solidification of neon at high pressure, the samples were under a quasi-hydrostatic condition that supports slight deviatoric stress. The measured pressure gradient from the center to the edge within the sample chamber was within $2 \mathrm{GPa}$ at the highest experimental pressure, indicated by the small shift of the neon peaks. In Figure $1 \mathrm{~b}$, the slight shift in neon (111) peak among the diffraction patterns of Fe-0C, Fe-0.31C, and Fe-1.37C clearly showed a non-hydrostatic condition in the samples chamber. Fe-1.37C was under the highest pressure $(33.1 \mathrm{GPa})$, and $\mathrm{Fe}-0 \mathrm{C}$, under the lowest pressure (31.7 GPa). In the meantime, we found that the lattice parameters for hcp-Fe-1.37C were the largest, while the lattice parameters for hcp-Fe- $0 \mathrm{C}$ were the smallest. The results indicated that the unit cell volume in the hcp structure increased with increasing carbon content under the same pressure (Figure 2a).

Tables S1 and S2 list the compression data for pure Fe, Fe-0.31C and Fe-1.37C in bct and the hcp structure. The pressure vs. volume data for hcp-Fe were used to fit to the third-order Birch-Murnaghan (3BM) equation of state (EoS) [25]:

$$
P=\frac{3 K_{0}}{2}\left[\left(\frac{V_{0}}{V}\right)^{\frac{7}{3}}-\left(\frac{V_{0}}{V}\right)^{\frac{5}{3}}\right]\left\{1+\frac{3}{4}\left(K_{0}^{\prime}-4\right)\left[\left(\frac{V_{0}}{V}\right)^{\frac{2}{3}}-1\right]\right\}
$$

where $P$ is the pressure (GPa); $V_{0}$ and $V$ are the unit-cell volumes $\left(\AA^{3}\right)$ at ambient conditions and high pressures, respectively; $K_{0}$ and $K_{0}{ }^{\prime}$ are the bulk modulus (GPa) and its pressure derivative at 
$300 \mathrm{~K}$, respectively. The fitting results for hcp-Fe were shown in Table 1 and the calculated isothermal compression curves for pure Fe, Fe-0.31C and Fe-1.37C in hcp phase are shown in Figure 2.

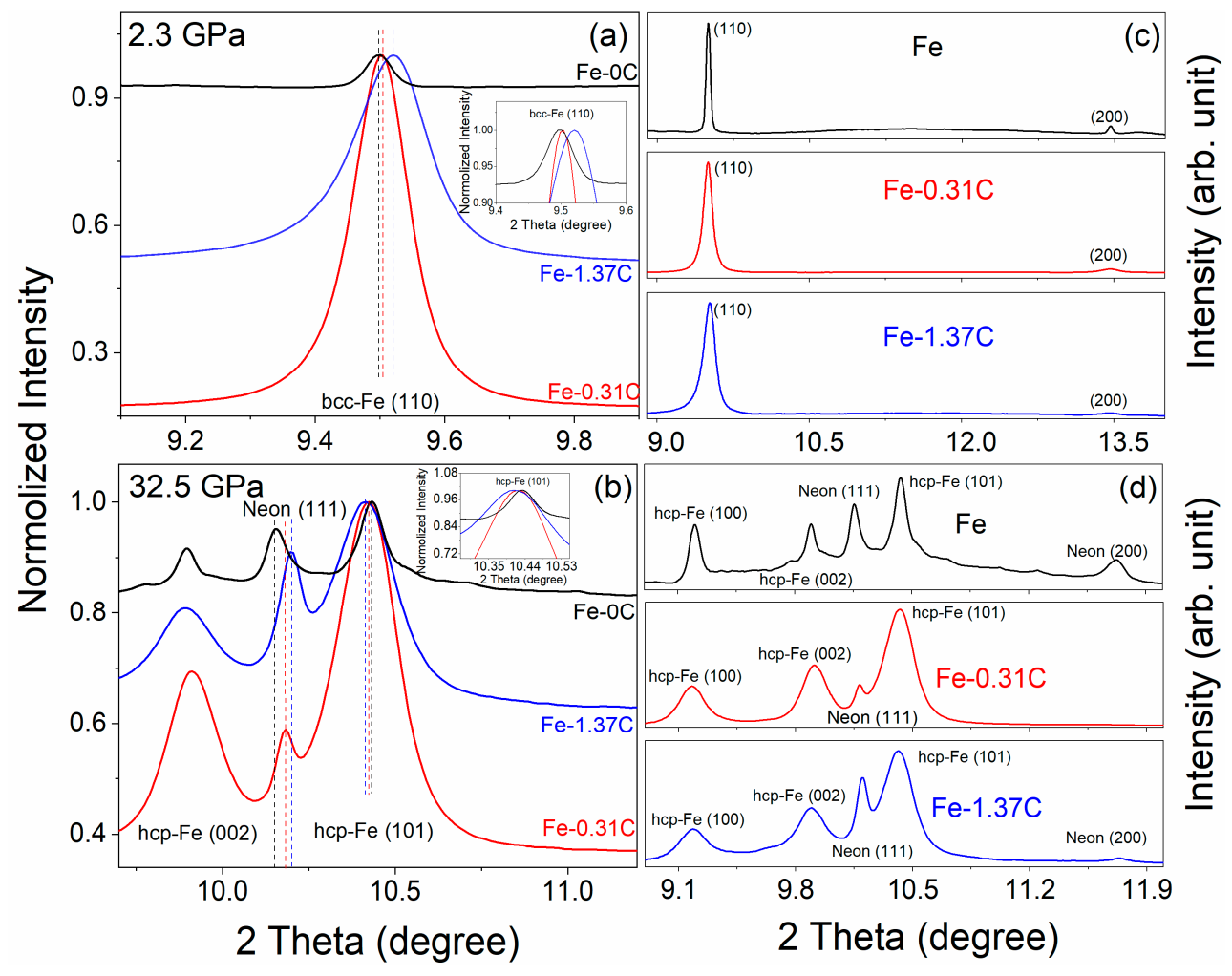

Figure 1. Representative $X$-ray diffraction (XRD) patterns for Fe-C samples in $(\mathbf{a}, \mathbf{c})$ selected peaks and full XRD patterns of the body-centered tetragonal (bct) structure at $2.3 \mathrm{GPa}$ and $(\mathbf{b}, \mathbf{d})$ selected peaks and full XRD pattern of hcp structure at $32.5 \mathrm{GPa}$, using $\mathrm{Au}$ as first-order pressure calibrant. Insert in (a) shows the zoom-in of the (110) peak of body-centered cubic (bcc)-Fe and (b) zoom-in of (101) and (002) peaks of hcp-Fe. Fe-0C (pure Fe), Fe-0.31C, Fe-1.37C, and Au are loaded in the same sample chamber using neon as the pressure medium. Black, red and blue curves are for Fe-0C, Fe-0.31C and Fe-1.37C, respectively. Neon (111) peak shift in each pattern for Fe-0C, Fe-0.31C and Fe-1.37C under 32.5 GPa indicates non-hydrostatic pressure in the sample chamber, with Fe-0C under lowest pressure, and $\mathrm{Fe}-1.37 \mathrm{C}$ under highest pressure. Vertical dash lines are plotted to show the center of each peak and for comparison of peak shift.

Figure 2a shows the compression curves for hcp-Fe, Fe-0.31C and Fe-1.37C up to $135 \mathrm{GPa}$. The trend of $P-V$ relation of pure Fe was in a good agreement with selected previous results [26-28] up to $135 \mathrm{GPa}$. The slightly offset of the absolute values of the volume for pure Fe may have been caused by using a different pressure calibrant or pressure medium in each experimental study. Because of the covariance between $V_{0}, K_{0}$ and $K_{0}{ }^{\prime}$, determination of $V_{0}$ of the high-pressure phase was difficult. In previous experimental studies, $V_{0}$ differed from 22.15 to $22.43 \AA^{3}{ }^{3}, K_{0}^{\prime}$ differed from 4.5 to 5.8 , and $K_{0}$ varied from 156 to $202 \mathrm{GPa}$ [26-32]. Here we used fixed $K_{0}{ }^{\prime}=4.79$ [27] for EoS fitting to systematically compare the EoS parameters for pure $\mathrm{Fe}, \mathrm{Fe}-0.31 \mathrm{C}$, and $\mathrm{Fe}-1.37 \mathrm{C}$ in this study. The volume at ambient $V_{0}$ calculated from $3 \mathrm{BM}$ EoS is 22.24(4), 22.26(3), and 22.44(3) $\AA^{3}$ for pure Fe, Fe-0.31C and Fe-1.37C, respectively. Without constraining the $K_{0}{ }^{\prime}$, we obtained $V_{0}=22.26(6), K_{0}=169.7(5.2)$ and $K_{0}{ }^{\prime}=5.19(16)$ for pure Fe, $V_{0}=22.37(4), K_{0}=168.9(4.8)$ and $K_{0}{ }^{\prime}=5.19(14)$ for Fe-0.31C, and $V_{0}=20.37(6), K_{0}=$ 182.2(4.2) and $K_{0}{ }^{\prime}=4.68(17)$ for Fe-1.37C. The fitting results were summarized in Table 1. The fitting results showed that $\mathrm{Fe}$ with more interstitial carbon would have a larger unit cell volume at the same pressure up to $\sim 135 \mathrm{GPa}$. The magnitude of increase remained the same over the pressure range of the study because the compression curves were almost parallel (Figure 2a). Figure S4 shows the unit 
cell volumes of the hcp phase at ambient conditions as a function of carbon content. Contrary to the volume-carbon content relation for the bcc phase in previous studies [2], the unit cell volume of hcp-Fe increased with increasing carbon content. This is the first experimental determination of the effect of carbon on the volume in the hcp structure that invalidates the common assumption that hcp and bcc iron have similar effect with respect to the interstitial carbon on the unit cell volume [2] as shown in Figure S4. The assumption may have significant effect on the calculated phase relations in the Fe-C system at high pressure, particularly in terms of the carbon solubility at very high pressure.

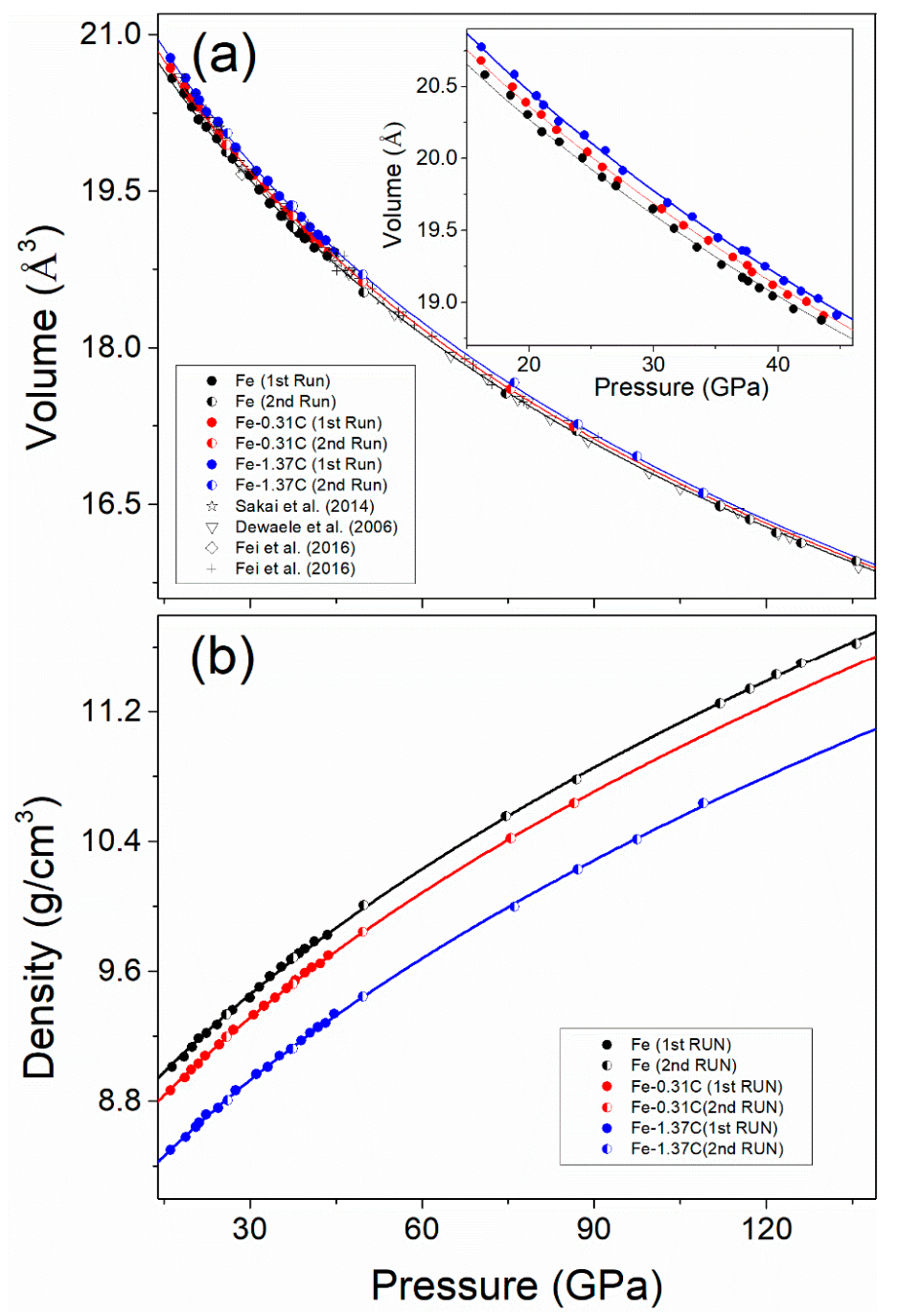

Figure 2. Compression curves for Fe-C samples. (a) Pressure and volume relation $(P-V)$ of hcp-Fe with $0,0.31$ and $1.37 \mathrm{wt} \%$ of carbon; (b) pressure and density relation $(P-\rho)$ of hcp-Fe with $0,0.31$ and 1.37 wt \% of carbon. Au was used as the pressure calibrant below $15 \mathrm{GPa}$, while neon peaks were used as the pressure calibrant above $15 \mathrm{GPa}$. Solid symbols represent data from the 1st RUN and half-open symbols represent data from the 2 nd RUN; the $P-V$ and $P-\rho$ relations were fitted using a third-order Birch-Murnaghan equation of state using combined data from 1st and 2nd RUN; black, red and blue curves are for Fe-0C, Fe-0.31C and Fe-1.37C, respectively.

The effect of interstitial carbon on the density is critical to estimate the carbon content in the Earth's inner core. The density as a function of pressure for hcp-Fe-C alloys are shown in Figure $2 b$. The results show that the density of Fe with $1.37 \mathrm{wt} \%$ of carbon was 5.6\% smaller than that of the pure Fe with hcp structure. Due to the volume expansion, interstitial carbon in the hcp structure enhanced the density contrast between the pure iron and Fe-C alloy. First principle calculations using the projector 
augmented wave (PAW) method also showed a density decrease with additional interstitial carbon in the Fe lattice. Huang et al. (2005) reported that the density of Fe with $1.3 \mathrm{wt} \%$ of carbon was $8.7 \%$ smaller than that of the pure Fe [33], while others yielded a smaller density decrease [16,34]. For example, Caracas (2017) showed that $1 \mathrm{wt} \%$ added carbon would decrease the density of hcp-Fe by about $2.8 \%$, while Li et al. (2018) showed that density of Fe with 1.4 wt \% carbon dropped by $2.4 \%[16,34]$.

Table 1. Elastic parameters for hcp-structure of Fe and Fe-C alloys in this and previous studies.

\begin{tabular}{|c|c|c|c|c|}
\hline Compositions & $V_{0}\left(\AA^{3}\right)$ & $\rho\left(\mathrm{g} / \mathrm{cm}^{3}\right)$ & $K_{0}(\mathrm{GPa})$ & $K_{0}^{\prime}$ \\
\hline \multicolumn{5}{|l|}{ This study } \\
\hline $\mathrm{Fe}$ & $22.24(4)$ & 8.392 & $187.3(1.3)$ & 4.79 (fixed) \\
\hline $\mathrm{Fe}$ & $22.26(6)$ & 8.332 & $169.7(5.2)$ & $5.19(16)$ \\
\hline $\mathrm{Fe}-0.31 \mathrm{C}$ & $22.26(3)$ & 8.242 & $182.8(1.0)$ & 4.79 (fixed) \\
\hline $\mathrm{Fe}-0.31 \mathrm{C}$ & $22.37(4)$ & 8.197 & $168.9(4.8)$ & $5.19(14)$ \\
\hline $\mathrm{Fe}-1.37 \mathrm{C}$ & $22.44(3)$ & 7.881 & $177.6(1.2)$ & 4.79 (fixed) \\
\hline Fe-1.37C & $20.37(6)$ & 7.895 & $182.2(4.2)$ & $4.68(17)$ \\
\hline \multicolumn{5}{|l|}{ Calculation $(\mathrm{Fe})$} \\
\hline Non-magnetic, Steinle-Neumann et al., (1999) [35] & 20.45 & 9.069 & 292 & 4.4 \\
\hline Steinle-Neumann et al., (1999) [35] & 21.10 & 8.790 & 209 & 5.2 \\
\hline Vočadlo et al., (2009) [36] & $20.86(4)$ & 8.891 & $237.8(4)$ & 4.8 \\
\hline Non-magnetic, Huang et al., (2005) [33] & 20.52 & 9.0385 & 289 & 4.46 \\
\hline Non-magnetic, Sha and Cohen (2010) [37] & 20.18 & 9.191 & 296 & 4.4 \\
\hline \multicolumn{5}{|l|}{ Calculation (Fe-1.3C) } \\
\hline Huang et al., 2005 [33] & 21.46 & 8.254 & 291 & 4.34 \\
\hline \multicolumn{5}{|l|}{ Experiments $(\mathrm{Fe})$} \\
\hline Fei et al. (2016) [27] & 22.427(fixed) & 8.270 (fixed) & $172.7(1.4)$ & 4.79 \\
\hline Fei et al. (2016) [27] & 22.185 & $8.360(24)$ & $191.4(5.3)$ & $4.52(8)$ \\
\hline Yamazaki et al. (2012) [31] & 22.15 & 8.373 & 202(7) & $4.5(2)$ \\
\hline Mao et al. (1990) [32] & $22.35(2)$ & 8.298 & $165(4)$ & $5.33(90)$ \\
\hline Dubrovinsky et al. (2000) [30] & 22.40 & 8.280 & $156(4)$ & 5.81 \\
\hline Boehler et al. (2008) [29] & $22.46(4)$ & 8.258 & $160(6)$ & $5.6(2)$ \\
\hline Dewaele et al. (2006) [28] & 22.43 & 8.269 & 165(fixed) & $4.97(4)$ \\
\hline
\end{tabular}

\section{Discussion}

\subsection{Carbon Substitution Mechanism}

As shown in the BSE images as well as the EMPA results (Figure S1), the carbon concentration in Fe samples was distributed homogenously, which indicated no other carbon-bearing phases existing in these samples. Thus, the carbon atoms added into Fe must be as an either interstitial or substitutional solid solution, which mostly depends on the atomic size factor. The interstitial solid solution is commonly found in Fe at ambient conditions because the atomic radius of Fe is $\sim 1.7$ times larger than the radius of carbon atoms. Carbon atoms fill the holes in the Fe lattice, occupying either octahedral sites (O-sites) or tetrahedral sites (T-sites) [22]. It has been suggested that interstitial carbon energetically prefers to occupy the O-sites in bcc-Fe instead of T-site at ambient conditions [38], where the $\mathrm{O}$-site is nearly twice larger than the T-site. Upon compression, the carbon atom may change its atomic environment in order to become energetically favorable. Theoretical calculations show that when the pressure exceeds $300 \mathrm{GPa}$, the interstitial carbon atoms in bcc-Fe energetically occupy the T-site instead of the O-site [33], which, however, has not been validated by experiments yet. Changing of the carbon occupancy-site may result in a change of physical properties of the carbon-bearing host. For example, carbon atoms would exert tensile strains on the surrounding crystal lattice to contract the lattice if the carbon atoms occupy the holes that are larger than atomic radius of the carbon atoms; conversely, if the carbon atoms are larger than the holes of the host $\mathrm{Fe}$, the carbon atoms would impose compressive strains in their vicinity, which would expand the volume of the lattice [22]. Increasing 
interstitial carbon content results in a decrease in unit-cell volume of bcc-Fe [2], which is consistent with the metallurgy study [22]. In this study, we showed that carbon expands both the $a$-axis and $c$-axis as well as the unit-cell volume of the hcp-Fe. The experimental results in this study, for the first time, revealed that carbon in hcp-Fe and bcc-Fe have an opposite effect on molar volume. The results may imply a change in the local environment for carbon atoms. One explanation could be that the carbon atoms enter the smaller T-site in hcp-Fe upon compression, resulting in the expansion of the unit cell because of the imposed compressive strains in the vicinity.

Another explanation for the carbon-induced volume change in hcp-Fe is that carbon atoms substitute the Fe atoms to form a substitutional solid solution instead of an interstitial solid solution. The substitutional solid solution commonly occurs in Fe-Si alloys. Both theoretical and experimental results show that Fe-Si alloys with hcp structure always have a larger unit-cell volume comparing to that of the pure hcp-Fe $[39,40]$. On the other hand, theoretical calculation shows that the hcp phase containing $\sim 6$ at. $\%$ of substitutional carbon becomes more stable than that with the same amount of interstitial carbon at pressures above $280 \mathrm{GPa}$ [33]. However, there is no report for the volume difference between the interstitial and substitutional carbon-bearing hcp-Fe. Further experimental analysis to detect the local environment of carbon in hcp-Fe have to be done to better understand the substitution mechanism of carbon in the hcp-Fe at high pressure.

Interstitial atoms in solid Fe would lock the dislocation movements and enhance the strength of the solid Fe, which has been extensively studied in the steel industry [41]. The ability of a metal to plastically deform depends on the ability of dislocations to move. In bcc-Fe, increasing interstitial carbon content increases the hardness and strength of the Fe in the carbon content range of $0.35-1.86 \mathrm{wt} \%$. Both tensile and compressive strain field interactions between dislocations and interstitial atoms result in the restriction of dislocation movements. The environmental change of carbon in $\mathrm{Fe}$, due to the phase transition from bcc to hcp could affect the movement of the dislocation, which would have significant implications for the deformation of Earth's inner core. Thus, further studies on the flow of dislocation for Fe with interstitial or substitutional carbon at high pressure would be necessary for the interpretation of the seismic anisotropy of Earth's interior.

\subsection{Anisotropic Axial Compressibility in the hop-Fe Phase}

As shown in Figure 3a, the axial compression data for $a$ and $c$ axes were fitted by the modified Birch-Murnaghan EoS equations:

$$
P=\frac{3 K_{x}}{2}\left[\left(\frac{x_{0}}{x}\right)^{7}-\left(\frac{x_{0}}{x}\right)^{5}\right]\left\{1+\frac{3}{2}\left(K_{x}^{\prime}-4\right)\left[\left(\frac{x_{0}}{x}\right)^{2}-1\right]\right\}
$$

where $x_{0}$ and $x$ represent the lattice constant $a$ or $c(\AA)$ at ambient and high-pressure conditions, $K_{x}$ and $K_{x}^{\prime}$ represent the axis compressibility (GPa) and its pressure derivative at $300 \mathrm{~K}$, respectively. To avoid the tradeoff between $K_{x}$ and $K_{x}^{\prime}$, we fixed $K_{x}^{\prime}$ at 4 in order to compare the $K_{x}$ systematically with carbon effects. The fitting yielded $\mathrm{Ka}$ for Fe-0C, Fe-0.31C and Fe-1.37C were 232.5(3.6), 223.0(2.7) and 210.4(3.7) $\mathrm{GPa}$, respectively. The result showed that the $a$ axis becomes more compressible with the increasing carbon content. For the $c$ axis, the fitting yielded $\mathrm{Kc}$ for Fe-0C, Fe-0.31C and Fe-1.37C was 224.6(3.3), 236.0(4.8) and 238.8(3.9) GPa, respectively, indicating that Fe-1.37C had the least compressible $c$ axis and $\mathrm{Fe}-\mathrm{OC}$ had the most compressible $c$ axis (Figure $3 \mathrm{~b}$ ). The results showed that the alloying carbon can increase the anisotropy of the hcp-Fe crystal structure.

It has been observed that strong seismic anisotropy exists in the Earth's inner core, with longitudinal waves travelling $\sim 3 \%$ faster along the polar axis than in the equatorial plane [42], which can be attributed to the preferred orientation of hcp-Fe with strong single-crystal elastic anisotropy. The question is widely debated on whether the $a$ axis or $c$ axis of hcp-Fe is parallel to the Earth's equatorial plane. Some studies reported that preferential alignment of the $a$ axis of hcp-Fe in the equatorial plane is the most plausible explanation for the observed compressional wave travel time anomalies or seismic anisotropy in the inner core $[43,44]$. However, polycrystalline plasticity simulations for pure hcp-Fe 
found that initially random polycrystal become preferentially oriented with their $c$ axes parallel to the equatorial plane, which can explain the observed seismic anisotropy [45]. Our results showed that $K a>K c$ for pure Fe with the $a$ axis being 3.4\% less compressible than the $c$ axis, which is also consistent with previous theoretical calculations with $C_{11}<C_{33}$ for hcp-Fe since $C_{11}$ and $C_{33}$ associated purely with compressional stresses and strains along the $a$ and $c$ axis, respectively [35]. With increasing carbon content to $1.37 \mathrm{wt} \%$, the axial compressibility reversed to $K c>K a$ with the $c$ axis being $13.5 \%$ less compressible than the $a$ axis, indicating that interstitial carbon in hcp-Fe could affect the direction of fast travelling waves in the Earth's inner core. It implied that if Fe with interstitial carbon presents in the Earth's core, the crystals preferentially oriented with the $a$ axis parallel to the equatorial plane would reconcile the seismic observation, which behaves in an opposite way compared to that of the pure Fe. On the other hand, carbon can enhance seismic anisotropy as increasing carbon content (Figure $2 b$ ) with the fastest axis of compressional wave velocity propagation along the $c$ axis. This contradicts the theoretical calculations, which showed that the fastest axis of compressional wave velocity propagation tilted away from the $c$ axis of Fe [16]. Further experiments should investigate the deformation mechanism in the hcp-Fe with interstitial carbon and the effects of interstitial substituted carbon on the seismic anisotropy in the Earth's core to resolve the contradiction.

The $c / a$ axial ratio of preferentially aligned crystals of hcp-Fe can be also related to the anisotropy of the Earth's inner core [46]. In theoretical calculations, the $c / a$ axial ratio serves as an input to calculate the elastic moduli or to model the core anisotropy, which has previously been done in the case of pure hcp-Fe $[35,36]$. Previous studies have shown that the $c / a$ ratio in hcp-Fe is closely related to pressure, temperature, and composition, which always increases with increasing temperature, but this effect is strongest at low pressure, and becomes weaker with increasing pressure. Some studies reported a trend of the $c / a$ ratio of hcp-Fe decreasing with increasing pressure [26-28,31], while others observed a weak trend or even no trend [32,47-49]. The value of $c / a$ ratio typically ranges from 1.60 to 1.61 over experimental pressures. Our results show that the $c / a$ ratio for pure Fe was $\sim 1.600-1.605$ with a decreasing trend up to $135 \mathrm{GPa}$ (Figure 3d), which is consistent with some of the previous studies. There have been studies on the effect of light elements on the $c / a$ ratio of the hcp-Fe [46]. For example, adding $2.8 \mathrm{~mol} \%$ sulfur to Fe-Ni alloy lowers the $c / a$ ratio by $\sim 0.005$ [50]. In this study, it has been observed that carbon also has an effect on the $c / a$ ratio of the hcp-Fe. The $c / a$ ratio for Fe- $0.31 \mathrm{C}$ and Fe-1.37C is $~ 1.603-1.607$ and 1.607 to 1.610 , respectively, and adding $1.37 \mathrm{wt} \%$ of carbon in the hcp-Fe would increase the $c / a$ ratio by $0.005-0.007$ at high pressures. The experimentally determined $c / a$ ratio for pure $\mathrm{Fe}, \mathrm{Fe}-0.31 \mathrm{C}$ and $\mathrm{Fe}-1.37 \mathrm{C}$ as a function of pressure has been linearly fitted to examine the pressure effect (Figure 3d). The results show that contrary to pure Fe, the $c / a$ ratio of Fe-C had an increasing trend with increasing pressure, which implied an enhanced anisotropy of hcp-Fe with increasing carbon content. 

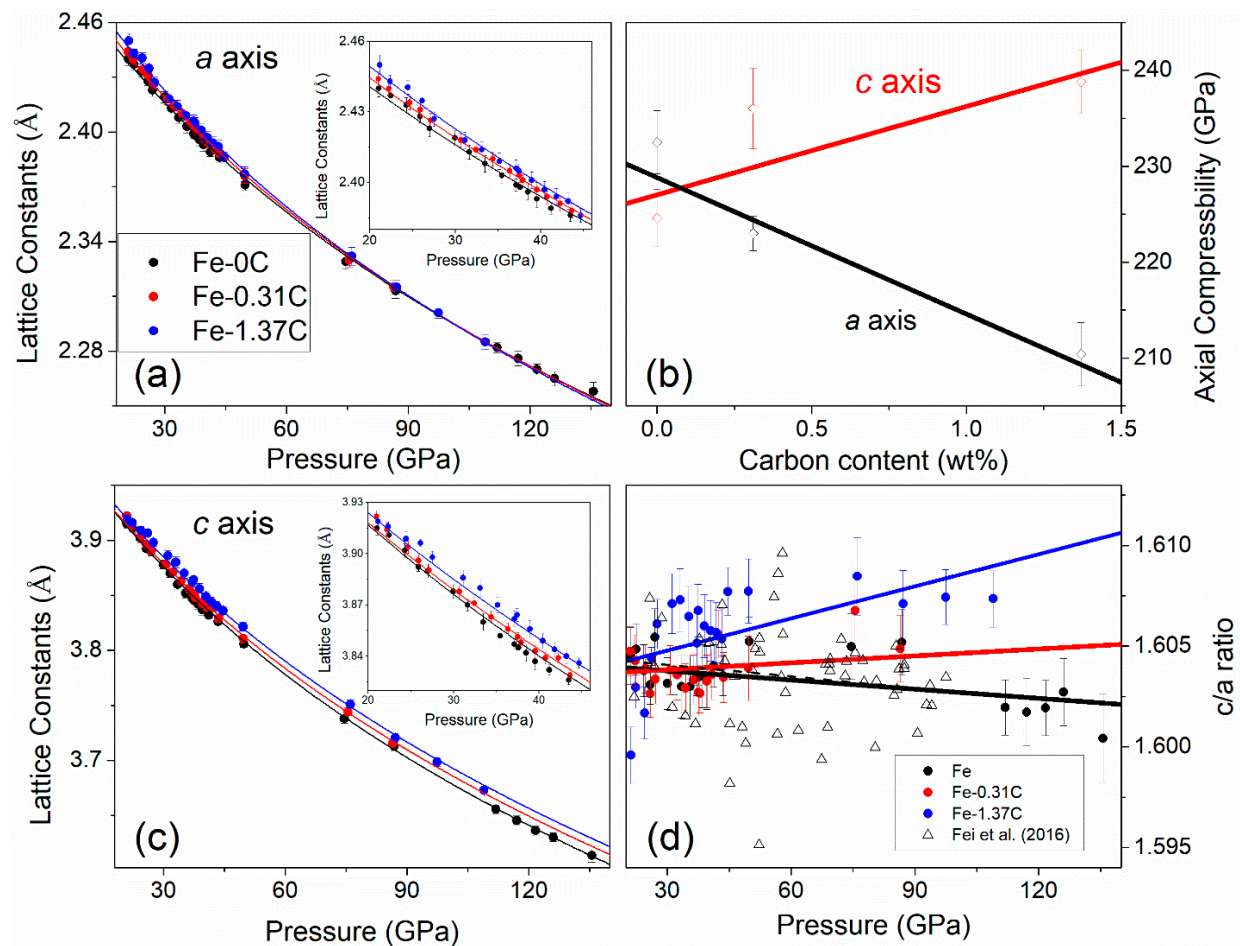

Figure 3. Compression of lattice constants $a$ and $c$ of the hcp-Fe with $0,0.31$ and $1.37 \mathrm{wt} \%$ of carbon. $(\mathbf{a}, \mathbf{c})$ The lattice constants of the $a$ and $c$ axes as a function of pressure; the experimental data were fitted by modified Birch-Murnaghan equation of state (EoS, see text for more detail); black, red and blue curves are for Fe-0C, Fe-0.31C and Fe-1.37C, respectively; inserts in $(\mathbf{a}, \mathbf{c})$ shows the zoomed plots from 20-45 GPa collected from 1st RUN; (b) the axial compressibility as function of carbon content; lines are linearly fitting of the data; (d) c/a ratio of hcp-Fe with $0,0.31$, and $1.37 \mathrm{wt} \%$ of carbon as a function of pressure; lines in $(\mathbf{b}, \mathbf{d})$ are linear fittings of the data.

\subsection{Density Deficit of Earth's Inner Core}

Incorporation of carbon in metallic iron can significantly reduce the density of hcp-Fe, providing a solution for the observed density deficit of the core if carbon is the dominant light element in the Earth's core. Due to the limited pressure and temperature range of the experimental data, thermoelastic modelling and extrapolation are needed to determine the carbon effect on the density under core conditions. Using the derived elastic moduli, the density of the hcp-Fe, Fe-0.31C, and Fe-1.37C at $300 \mathrm{~K}$ together with previously determined thermoelastic parameters, we can model the density of carbon-bearing Fe along an adiabatic geotherm by determining the thermal pressures based on the Debye model:

$$
P_{\text {th }}=P-P_{300 \mathrm{~K}}=\frac{\gamma_{v i b}}{V} \int_{300}^{T} C_{V, v i b} d T+\frac{\gamma_{e}}{V} \int_{300}^{T} C_{V e} d T
$$

where $P_{300 \mathrm{~K}}$ is the reference pressure at $300 \mathrm{~K}$ from this study, $V$ is the unit cell volume $\left(\AA^{3}\right), \gamma_{\text {vib }}$ is the vibrational Grüneisen parameter, $\gamma_{e}$ is the electronic Grüneisen parameter, $C_{V, \text { vib }}$ is the vibrational specific heat $\left(\mathrm{Jkg}^{-1} \mathrm{~K}^{-1}\right)$ and $C_{V e}$ is the electronic specific heat $\left(\mathrm{Jkg}^{-1} \mathrm{~K}^{-1}\right)$. Because there are no measured thermoelastic parameters for carbon-bearing hcp-Fe, we adopted the parameters (Table S3) from the pure hcp-Fe derived from combined static $P-V-T$ data up to $200 \mathrm{GPa}$ and $1795 \mathrm{~K}$ [27] and dynamic Hugoniot data [51] for the calculations. To test the reliability of the modelling, we calculated the density profile for pure-Fe along the adiabatic geotherm with a fixed ICB temperature of 6000 $\mathrm{K}\left(\mathrm{T}_{\mathrm{ICB}}=6000 \mathrm{~K}\right)$, which is comparable to that reported by Fei et al. (2016) within the experimental uncertainties [27] (Figure 4). The calculated density profile for Fe-1.37C plotted below that of the 
Preliminary Reference Earth Model (PREM) for the inner core, indicating that less than 1.37 wt \% carbon in the inner core was required to explain the density deficit.

With the established pressure-density relations for pure Fe, Fe- $0.31 \mathrm{C}$ and $\mathrm{Fe}-1.37 \mathrm{C}$ along the geotherm with the fixed $\mathrm{T}_{\mathrm{ICB}}$ of 5000,6000 and $7000 \mathrm{~K}$, we can estimate the carbon content that can reconciled the density deficit in the Earth's inner core depending on the thermal profile of the core. Using the Debye thermoelastic model $(f)$ as described in Equation (3), we calculated the density of pure $\mathrm{Fe}\left(d_{F e}\right), \mathrm{Fe}-0.31 \mathrm{C}\left(d_{F e-0.31 \mathrm{C}}\right)$, and Fe-1.37C $\left(d_{F e-1.37 \mathrm{C}}\right)$ as a function of pressure alone the geotherm:

$$
\begin{gathered}
d_{F e}=f_{F e}(P, T), \\
d_{F e-0.31 C}=(1-0.31 \%) d_{F e}+0.31 \% \Delta d_{0.31} \\
d_{F e-1.37 C}=(1-1.37 \%) d_{F e}+1.37 \% \Delta d_{1.37}
\end{gathered}
$$

where $\Delta d_{0.31}$ and $\Delta d_{1.37}$ represent density deviation from the density profile of pure Fe for Fe-0.31C and Fe-1.37C, respectively, which represent the carbon effect on the density of hcp-Fe. Using the PREM density profile $\left(d_{P R E M}\right)$ as the constraint, we performed least-squares regression to determine the carbon content through:

$$
d_{P R E M}=(1-x) d_{F e}+x \Delta d_{x}
$$

where $x$ is the predicted carbon content in the Earth inner core. The results yielded the carbon content $x=1.30(8) \%$ with $\mathrm{T}_{\mathrm{ICB}}=5000 \mathrm{~K}, x=0.95(5) \%$ with $\mathrm{T}_{\mathrm{ICB}}=6000 \mathrm{~K}$, and $x=0.43(13) \%$ with $\mathrm{T}_{\mathrm{ICB}}=$ $7000 \mathrm{~K}$, which provided the best match to the density profile of PREM at inner core condition (Figure 4). Our results showed that on the basis of density constraint alone, incorporation of $0.43-1.30 \mathrm{wt} \%$ carbon in the inner core is sufficient to account for the inner core density deficit depending on the thermal profile. Previous results on the melting of Fe-C binary system showed that the carbon solubility in the solid Fe would remain a constant value of $1 \mathrm{wt} \%$ up to $255 \mathrm{GPa}$ [6]. If this is the case and carbon is the only light element in the Earth's inner core, $\sim 6000 \mathrm{~K}$ would be a good estimation of the inner core boundary temperature.

Considering the carbon partitioning between liquid and solid Fe, $D_{C}^{\text {liquid/solid }} \approx 2$, in the Fe-C binary system at inner core boundary conditions [2], the outer core would contain $\sim 0.86-2.60 \mathrm{wt} \%$ carbon based solely on partitioning data. The determined carbon content in the outer core would be less than the recently determined eutectic composition of Fe-C system, which is $3 \mathrm{wt} \%$ carbon at the inner core boundary [6]. Thus, we would expect a hcp-Fe phase with dissolved carbon instead of Fe carbide crystallize at the inner core boundary. Previous studies have suggested that $1.7-3.8 \mathrm{wt} \%$ carbon is necessary to account for the density deficit [52,53] in the Earth's outer core, which is comparable with our estimations for the carbon content in the Earth's outer core. The amount of carbon would be reduced if other light elements also exist in the inner core. Sound velocity could provide additional constraint on the carbon content in the core. Theoretical calculations show that interstitial carbon increases compressional seismic wave velocity, while decreases shear wave velocity [16]. On the other hand, Li et al. (2018) inferred that the decreasing sound velocities and density of hcp-Fe with dissolved carbon cannot simultaneously match PREM, which has not been validated experimentally [34]. Thus, the sound velocity measurement of hcp-Fe with dissolved carbon, not only iron carbides, at high pressures would be essential for understanding the role of carbon in the solid inner core. 

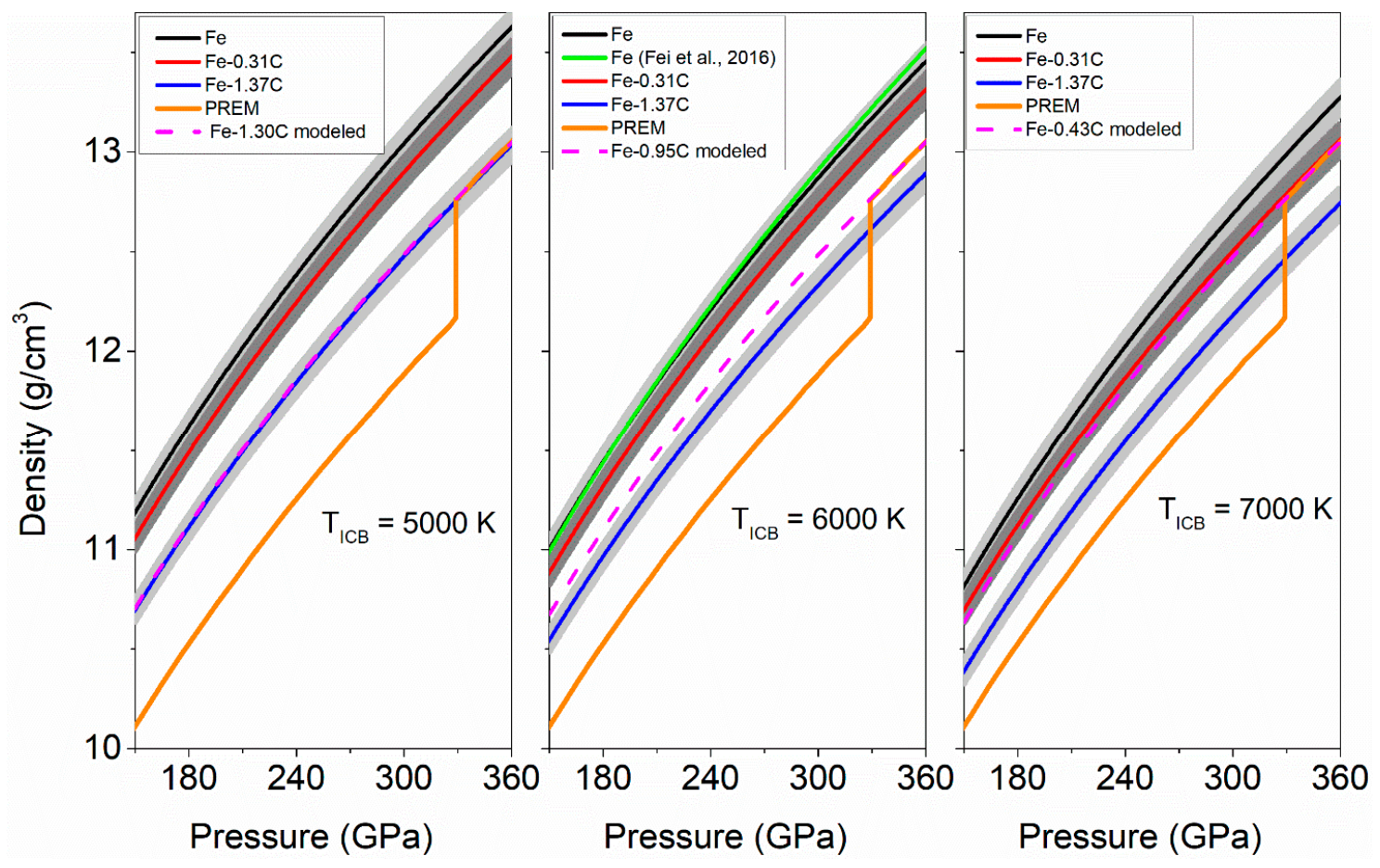

Figure 4. The calculated densities of hcp-Fe with $0,0.31$ and $1.37 \mathrm{wt} \%$ of carbon along the adiabatic geotherm with fixed temperatures of 5000, 6000 and $7000 \mathrm{~K}$ at the inner core boundary ( $\mathrm{T}_{\mathrm{ICB}}$ ), compared with density profile of the core from PREM. Black, red and blue solid lines represent the density profile of pure $\mathrm{Fe}, \mathrm{Fe}-0.31 \mathrm{C}$ and $\mathrm{Fe}-1.37 \mathrm{C}$, respectively. The grey shaded area represents the propagated uncertainties for extrapolated density. The green line is the literature data for pure Fe along the adiabatic geotherm with a fixed temperature of $6000 \mathrm{~K}$ at the inner core boundary (Fei et al., 2016 [27]). The orange line represents the density profile of PREM seismic model. The dashed magenta line represent the modeled densities of Fe contains 1.30(7) 0.95 (5) and 0.43(10) wt \% carbon at 5000, 6000, and $7000 \mathrm{~K}$ of $\mathrm{T}_{\mathrm{ICB}}$ that can reconcile the density profile of the PREM.

Supplementary Materials: The following are available online at http://www.mdpi.com/2075-163X/9/12/720/s1, Figure S1: Carbon analysis for recovered Fe-C samples by electron microprobe; Figure S2: Back scattered electron images for unsuccessful synthesis runs; Figure S3: The length of lattice constant a as a function of pressure for Fe, Fe-0.31C, and Fe-1.37C; Figure S4: Unit cell volume of bcc- and hcp-Fe as a function of carbon content at ambient condition; Table S1: Compression data for pure-Fe, Fe-0.31C and Fe-1.37C in bct structure using Au as the pressure calibrant; Table S2: Compression data for pure-Fe, Fe-0.31C and $\mathrm{Fe}-1.37 \mathrm{C}$ in hcp phases using Ne as the pressure calibrant; Table S3: Thermoelastic parameters used for modeling the density of hcp-Fe, Fe-0.31C and Fe-1.37C along an adiabatic geotherm with $\mathrm{T}_{\mathrm{ICB}}=5000,6000$ and $7000 \mathrm{~K}$.

Author Contributions: Conceptualization, J.Y. and Y.F.; methodology, J.Y., Y.F., E.G. and V.B.P.; formal analysis, J.Y. and X.H.; writing, J.Y. and Y.F.; all authors contribute review and editing.

Funding: This work was funded by an National Science Foundation (NSF) grant (EAR-1619868 to Y.F.). GSECARS was supported by the National Science Foundation (EAR-0622171) and U.S. Department of Energy (DE-FG02-94ER14466) under contract DE-AC02-06CH11357. APS was supported by Department of Energy Basic Energy Sciences Program (DOE-BES), under contract DE-AC02-0611357.

Acknowledgments: The authors thank Zhixue Du for their assistance with the X-ray diffraction experiments at 13ID-D, GSECARS. The authors also thank Megan Duncan and Emma Bullock for their guidance of carbon analysis.

Conflicts of Interest: The authors declare no conflict of interest.

\section{References}

1. Buchwald, V.F. Handbook of Iron Meteorites: Their History, Distribution, Composition, and Structure; University of California Press: Berkeley, CA, USA, 1975. 
2. Fei, Y.; Brosh, E. Experimental study and thermodynamic calculations of phase relations in the Fe-C system at high pressure. Earth Planet. Sci. Lett. 2014, 408, 155-162. [CrossRef]

3. Wood, B.J. Carbon in the core. Earth Planet. Sci. Lett. 1993, 117, 593-607. [CrossRef]

4. Prescher, C.; Dubrovinsky, L.; Bykova, E.; Kupenko, I.; Glazyrin, K.; Kantor, A.; McCammon, C.; Mookherjee, M.; Nakajima, Y.; Miyajima, N. High Poisson's ratio of Earth's inner core explained by carbon alloying. Nat. Geosci. 2015, 8, 220. [CrossRef]

5. Lord, O.; Walter, M.; Dasgupta, R.; Walker, D.; Clark, S. Melting in the Fe-C system to 70 GPa. Earth Planet. Sci. Lett. 2009, 284, 157-167. [CrossRef]

6. Mashino, I.; Miozzi, F.; Hirose, K.; Morard, G.; Sinmyo, R. Melting experiments on the Fe-C binary system up to 255 GPa: Constraints on the carbon content in the Earth's core. Earth Planet. Sci. Lett. 2019, 515, 135-144. [CrossRef]

7. Chen, B.; Li, Z.; Zhang, D.; Liu, J.; Hu, M.Y.; Zhao, J.; Bi, W.; Alp, E.E.; Xiao, Y.; Chow, P. Hidden carbon in Earth's inner core revealed by shear softening in dense $\mathrm{Fe}_{7} \mathrm{C}_{3}$. Proc. Natl. Acad. Sci. USA 2014, 111, 17755-17758. [CrossRef]

8. Liu, J.; Li, J.; Ikuta, D. Elastic softening in $\mathrm{Fe}_{7} \mathrm{C}_{3}$ with implications for Earth's deep carbon reservoirs. J. Geophys. Res. Solid Earth 2016, 121, 1514-1524. [CrossRef]

9. Belonoshko, A.B.; Ahuja, R.; Johansson, B. Stability of the body-centred-cubic phase of iron in the Earth's inner core. Nature 2003, 424, 1032. [CrossRef]

10. Belonoshko, A.B.; Lukinov, T.; Fu, J.; Zhao, J.; Davis, S.; Simak, S.I. Stabilization of body-centred cubic iron under inner-core conditions. Nat. Geosci. 2017, 10, 312. [CrossRef]

11. Tateno, S.; Hirose, K.; Ohishi, Y.; Tatsumi, Y. The structure of iron in Earth's inner core. Science 2010, 330, 359-361. [CrossRef]

12. Mao, H.-k.; Shu, J.; Shen, G.; Hemley, R.J.; Li, B.; Singh, A.K. Elasticity and rheology of iron above 220 GPa and the nature of the Earth's inner core. Nature 1998, 396, 741. [CrossRef]

13. Stixrude, L.; Cohen, R. High-pressure elasticity of iron and anisotropy of Earth's inner core. Science 1995, 267, 1972-1975. [CrossRef] [PubMed]

14. Karato, S.-I. Inner core anisotropy due to the magnetic field-Induced preferred orientation of iron. Science 1993, 262, 1708-1711. [CrossRef] [PubMed]

15. Song, X. Anisotropy of the Earth's inner core. Rev. Geophys. 1997, 35, 297-313. [CrossRef]

16. Caracas, R. The influence of carbon on the seismic properties of solid iron. Geophys. Res. Lett. 2017, 44, 128-134. [CrossRef]

17. Dasgupta, R.; Walker, D. Carbon solubility in core melts in a shallow magma ocean environment and distribution of carbon between the Earth's core and the mantle. Geochim. Cosmochim. Acta 2008, 72, 4627-4641. [CrossRef]

18. Robaut, F.; Crisci, A.; Durand-Charre, M.; Jouanne, D. Practical aspects of carbon content determination in carburized steels by EPMA. Microsc. Microanal. 2006, 12, 331-334. [CrossRef]

19. Fei, Y.; Ricolleau, A.; Frank, M.; Mibe, K.; Shen, G.; Prakapenka, V. Toward an internally consistent pressure scale. Proc. Natl. Acad. Sci. USA 2007, 104, 9182-9186. [CrossRef]

20. Prescher, C.; Prakapenka, V.B. DIOPTAS: A program for reduction of two-dimensional X-ray diffraction data and data exploration. High Press. Res. 2015, 35, 223-230. [CrossRef]

21. Toby, B.H. EXPGUI, a graphical user interface for GSAS. J. Appl. Crystallogr. 2001, 34, 210-213. [CrossRef]

22. Callister, W.D.; Rethwisch, D.G. Materials Science and Engineering: An Introduction; John Wiley \& Sons: New York, NY, USA, 2007; Volume 7.

23. Walker, D.; Dasgupta, R.; Li, J.; Buono, A. Nonstoichiometry and growth of some Fe carbides. Contrib. Mineral. Petrol. 2013, 166, 935-957. [CrossRef]

24. Lu, Y.; Yu, H.; Cai, X.; Rong, Y.; Sisson, R.D. Martensite lattice parameter measured by modern X-ray Diffraction in Fe-C alloy. In Proceedings of the 23rd International Federation of Heat Treatment and Surface Engineering Congress (IFHTSE 2016), Savannah, GA, USA, 18-21 April 2016.

25. Birch, F. Finite elastic strain of cubic crystals. Phys. Rev. 1947, 71, 809. [CrossRef]

26. Sakai, T.; Takahashi, S.; Nishitani, N.; Mashino, I.; Ohtani, E.; Hirao, N. Equation of state of pure iron and $\mathrm{Fe}_{0.9} \mathrm{Ni}_{0.1}$ alloy up to 3 Mbar. Phys. Earth Planet. Inter. 2014, 228, 114-126. [CrossRef]

27. Fei, Y.; Murphy, C.; Shibazaki, Y.; Shahar, A.; Huang, H. Thermal equation of state of hcp-iron: Constraint on the density deficit of Earth's solid inner core. Geophys. Res. Lett. 2016, 43, 6837-6843. [CrossRef] 
28. Dewaele, A.; Loubeyre, P.; Occelli, F.; Mezouar, M.; Dorogokupets, P.I.; Torrent, M. Quasihydrostatic equation of state of iron above 2 Mbar. Phys. Rev. Lett. 2006, 97, 215504. [CrossRef] [PubMed]

29. Boehler, R.; Santamaría-Pérez, D.; Errandonea, D.; Mezouar, M. Melting, density, and anisotropy of iron at core conditions: New X-ray measurements to 150 GPa. J. Phys. Conf. Ser. 2008, 121, 022018. [CrossRef]

30. Dubrovinsky, L.; Saxena, S.; Tutti, F.; Rekhi, S.; LeBehan, T. In situ X-ray study of thermal expansion and phase transition of iron at multimegabar pressure. Phys. Rev. Lett 2000, 84, 1720. [CrossRef]

31. Yamazaki, D.; Ito, E.; Yoshino, T.; Yoneda, A.; Guo, X.; Zhang, B.; Sun, W.; Shimojuku, A.; Tsujino, N.; Kunimoto, T. P-V-T equation of state for $\varepsilon$-iron up to $80 \mathrm{GPa}$ and $1900 \mathrm{~K}$ using the Kawai-type high pressure apparatus equipped with sintered diamond anvils. Geophys. Res. Lett. 2012, 39. [CrossRef]

32. Mao, H.; Wu, Y.; Chen, L.; Shu, J.; Jephcoat, A.P. Static compression of iron to $300 \mathrm{GPa}$ and $\mathrm{Fe}_{0.8} \mathrm{Ni}_{0.2}$ alloy to 260 GPa: Implications for composition of the core. J. Geophys. Res. Solid Earth 1990, 95, 21737-21742. [CrossRef]

33. Huang, L.; Skorodumova, N.; Belonoshko, A.B.; Johansson, B.; Ahuja, R. Carbon in iron phases under high pressure. Geophys. Res. Lett. 2005, 32. [CrossRef]

34. Li, Y.; Vočadlo, L.; Brodholt, J.P. The elastic properties of hcp-Fe alloys under the conditions of the Earth's inner core. Earth Planet. Sci. Lett. 2018, 493, 118-127. [CrossRef]

35. Steinle-Neumann, G.; Stixrude, L.; Cohen, R.E. First-principles elastic constants for the hcp transition metals Fe, Co, and Re at high pressure. Phys. Rev. B 1999, 60, 791. [CrossRef]

36. Vočadlo, L.; Dobson, D.P.; Wood, I.G. Ab initio calculations of the elasticity of hcp-Fe as a function of temperature at inner-core pressure. Earth Planet. Sci. Lett. 2009, 288, 534-538. [CrossRef]

37. Sha, X.; Cohen, R. First-principles thermal equation of state and thermoelasticity of hcp Fe at high pressures. Phys. Rev. B 2010, 81, 094105. [CrossRef]

38. Williamson, G.; Smallman, R. X-ray evidence for the interstitial position of carbon in $\alpha$-iron. Acta Crystallogr. 1953, 6, 361-362. [CrossRef]

39. Komabayashi, T.; Pesce, G.; Morard, G.; Antonangeli, D.; Sinmyo, R.; Mezouar, M. Phase transition boundary between fcc and hcp structures in Fe-Si alloy and its implications for terrestrial planetary cores. Am. Mineral. J. Earth Planet. Mater. 2019, 104, 94-99. [CrossRef]

40. Fischer, R.A.; Campbell, A.J.; Caracas, R.; Reaman, D.M.; Heinz, D.L.; Dera, P.; Prakapenka, V.B. Equations of state in the Fe-FeSi system at high pressures and temperatures. J. Geophys. Res. Solid Earth 2014, 119, 2810-2827. [CrossRef]

41. Goldschmid, H.J. Interstitial Alloys; Springer: Berlin, Germany, 2013.

42. Morelli, A.; Dziewonski, A.M.; Woodhouse, J.H. Anisotropy of the inner core inferred from PKIKP travel times. Geophys. Res. Lett. 1986, 13, 1545-1548. [CrossRef]

43. Lincot, A.; Merkel, S.; Cardin, P. Is inner core seismic anisotropy a marker for plastic flow of cubic iron? Geophys. Res. Lett. 2015, 42, 1326-1333. [CrossRef]

44. Antonangeli, D.; Merkel, S.; Farber, D.L. Elastic anisotropy in hcp metals at high pressure and the sound wave anisotropy of the Earth's inner core. Geophys. Res. Lett. 2006, 33. [CrossRef]

45. Buffett, B.; Wenk, H.-R. Texturing of the Earth's inner core by Maxwell stresses. Nature 2001, 413, 60. [CrossRef] [PubMed]

46. Fischer, R.A.; Campbell, A.J. The axial ratio of hcp Fe and Fe-Ni-Si alloys to the conditions of Earth's inner core. Am. Mineral. 2015, 100, 2718-2724. [CrossRef]

47. Jephcoat, A.P.; Mao, H.; Bell, P.M. Static compression of iron to $78 \mathrm{GPa}$ with rare gas solids as pressure-transmitting media. J. Geophys. Res. Solid Earth 1986, 91, 4677-4684. [CrossRef]

48. Ma, Y.; Somayazulu, M.; Shen, G.; Mao, H.-K.; Shu, J.; Hemley, R.J. In situ X-ray diffraction studies of iron to Earth-core conditions. Phys. Earth Planet. Inter. 2004, 143, 455-467. [CrossRef]

49. Ono, S.; Kikegawa, T.; Hirao, N.; Mibe, K. High-pressure magnetic transition in hcp-Fe. Am. Mineral. 2010, 95, 880-883. [CrossRef]

50. Sakai, T.; Ohtani, E.; Kamada, S.; Terasaki, H.; Hirao, N. Compression of $\mathrm{Fe}_{88.1} \mathrm{Ni}_{9.1} \mathrm{~S}_{2.8}$ alloy up to the pressure of Earth's inner core. J. Geophys. Res. Solid Earth 2012, 117. [CrossRef]

51. Brown, J.; Fritz, J.; Hixson, R. Hugoniot data for iron. J. Appl. Phys. 2000, 88, 5496-5498. [CrossRef] 
52. Nakajima, Y.; Imada, S.; Hirose, K.; Komabayashi, T.; Ozawa, H.; Tateno, S.; Tsutsui, S.; Kuwayama, Y.; Baron, A.Q. Carbon-depleted outer core revealed by sound velocity measurements of liquid iron-carbon alloy. Nat. Commun. 2015, 6, 8942. [CrossRef]

53. Morard, G.; Andrault, D.; Antonangeli, D.; Nakajima, Y.; Auzende, A.; Boulard, E.; Cervera, S.; Clark, A.; Lord, O.; Siebert, J. Fe-FeO and $\mathrm{Fe}-\mathrm{Fe}_{3} \mathrm{C}$ melting relations at Earth's core-mantle boundary conditions: Implications for a volatile-rich or oxygen-rich core. Earth Planet. Sci. Lett. 2017, 473, 94-103. [CrossRef]

(C) 2019 by the authors. Licensee MDPI, Basel, Switzerland. This article is an open access article distributed under the terms and conditions of the Creative Commons Attribution (CC BY) license (http://creativecommons.org/licenses/by/4.0/). 